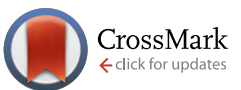

Cite this: Lab Chip, 2016, 16, 1157

Received 11th February 2016, Accepted 29th February 2016

DOI: 10.1039/c6lc00197a

\section{Calcium oxalate precipitation by diffusion using laminar microfluidics: toward a biomimetic model of pathological microcalcifications $\dagger$}

\author{
G. Laffite, ${ }^{\text {ab }}$ C. Leroy, ${ }^{b}$ C. Bonhomme, ${ }^{b}$ L. Bonhomme-Coury, ${ }^{b}$ E. Letavernier, ${ }^{c}$ \\ M. Daudon, ${ }^{c}$ V. Frochot, ${ }^{C}$ J. P. Haymann, ${ }^{C}$ S. Rouzière, ${ }^{d}$ I. T. Lucas, ${ }^{e}$ D. Bazin, ${ }^{\text {bd }}$ \\ F. Babonneau ${ }^{\mathrm{b}}$ and A. Abou-Hassan*a
}

www.rsc.org/loc

The effect of mixing calcium and oxalate precursors by diffusion at miscible liquid interfaces on calcium oxalate crystalline phases, and in physiological conditions (concentrations and flow rates), is studied using a microfluidic channel. This channel has similar dimensions as the collection duct in human kidneys and serves as a biomimetic model in order to understand the formation of pathological microcalcifications.

During the last decades, epidemiological studies have suggested an increased frequency of kidney stone (KS) diseases affecting up to $10 \%$ of the industrialized population. ${ }^{1}$ Nowadays, most of the kidney stones are made of calcium oxalate (CaOx) crystalline aggregates. ${ }^{2}$ Due to these alarming perspectives, a large number of investigations have been dedicated to in vitro crystallisation methods. ${ }^{3}$ Their aim is to study the impact of local physico-chemical variations on the formation of the stable CaOx monohydrated form whewellite (calcium oxalate monohydrate, COM) or the less stable $\mathrm{CaOx}$ dihydrated form weddellite (calcium oxalate dihydrate, COD). However, some studies pointed out the fact that the morphology and the nature of $\mathrm{CaOx}$ phases could be significantly affected by the flow procedure used for precipitation. $^{4}$

\footnotetext{
${ }^{a}$ Sorbonne Universités, UPMC Univ Paris 06, UMR 8234 CNRS, Laboratoire Physico-chimie des Electrolytes et Nanosystèmes Interfaciaux (PHENIX), 4 place Jussieu - case 51, F-75005, Paris, France. E-mail: ali.abou_hassan@upmc.fr

${ }^{b}$ Sorbonne Universités, UPMC Univ Paris 06, CNRS, Collège de France, Laboratoire de Chimie de la Matière Condensée de Paris (LCMCP), 11 Place Marcelin Berthelot, Bat D, F-75005, Paris, France

${ }^{c}$ Sorbonne Universités, UPMC Univ Paris 06, AP-HP, INSERM, Service d'Explorations Fonctionnelles, Hôpital Tenon, 4 rue de la Chine, 75020, Paris, France

${ }^{d}$ Laboratoire de Physique des Solides, CNRS UMR 8502, Université Paris-Sud, Bât 510, 91405, Orsay, France

${ }^{e}$ Sorbonne Universités, UPMC Univ Paris 06, UMR 8235 CNRS, Laboratoire Interfaces et Systèmes Electrochimiques (LISE), 4 place Jussieu - case 133, F75005, Paris, France

$\dagger$ Electronic supplementary information (ESI) available: Chemicals, microfluidic device fabrication, COMSOL simulations, and experimental results (FT-IR and XRD). See DOI: 10.1039/c6lc00197a
}

Indeed, CaOx crystals leading to stones appear at the end of the collecting duct, which is the most distal part of the kidney tubular structure responsible for urine concentration. $^{3 c}$ The human diuresis (urine secretion) varies

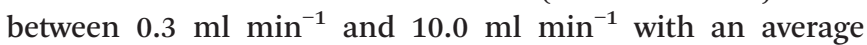
nephron (urine forming unit) number ranging from approximately 200000 to more than 2.5 million in human kidneys. ${ }^{5}$ Considering that about 10 nephrons give birth to one single collecting duct, the flow rate inside one collecting duct is around $10.0 \mathrm{nl} \mathrm{min}{ }^{-1}$ under a standard diet in human beings. Such a flow rate is in the order of magnitude to those injected in microsystems. Moreover in humans the diameter of a collecting duct is $\approx 100 \mu \mathrm{m}$ (which can be easily mimicked using microchannels). ${ }^{6}$ It follows that urine flow is laminar in collecting ducts. ${ }^{7}$ Finally, it is anticipated that KS growth kinetics should be mainly controlled by diffusion gradients in a state of continuous flow with a constant replenishment of oxalate and calcium solutions (i.e. maintaining a constant supersaturation (SS) state). Such gradients can be easily reproduced using a microfluidic channel. In conclusion, it appears clear that a biomimetic model using microfluidics can be built to mimic the conditions for $\mathrm{CaOx}$ precipitation in kidneys.

Due to their small dimensions, flows are laminar in continuous microfluidic devices and mixing occurs by molecular diffusion at the dynamic interfaces of two or several miscible liquids. Previously, the laminarity of the flow has been used to generate structures by patterning of metals, organic polymers, inorganic crystals, and ceramics at interfaces. ${ }^{8}$ Moreover a laminar microfluidic device offers great potential to mimic organs on chips. ${ }^{9}$ For instance, Wei et al. ${ }^{10}$ developed a bio-inspired kidney model to understand the molecular and pharmacological basis of calcium phosphate stone formation in the epithelial or other similar cellular microenvironments. To the best of our knowledge, investigating the effect of diffusive mixing on the crystalline phases of calcium oxalate obtained at miscible liquid-liquid dynamical interfaces in a microfluidic device and in the physiological 
conditions (concentrations and flow rates) has never been reported. Such a biomimetic model is important in order to understand the formation of pathological microcalcifications.

The principles of our experimental approach are illustrated in Fig. 1a. The upper part of the device is composed of a "Y-shaped" polydimethylsiloxane (PDMS) microchannel where calcium and oxalate precursor solutions are injected through separated inlets in the microchannel, mixed by molecular interdiffusion at the interface and resulting in the precipitation of $\mathrm{CaOx}$ crystals in the interdiffusion zone.

The initial mold used for PDMS replication is fabricated using a standard soft lithography process described in ESI. $\dagger$ The microchannel dimensions are fixed to $5 \mathrm{~cm}$ in length, $100 \mu \mathrm{m}$ in width and $100 \mu \mathrm{m}$ in depth to correctly mimic a collecting duct in human kidneys. In contrast to the standard elastomer-based device fabrication process, we selected a reversible sealing assembly ${ }^{11}$ in order to remove the upper PDMS part from the flat support substrate. For that purpose, we manufactured a specific metallic holder to maintain mechanically a homogeneous compression between both parts of the microchannel and to avoid any leakage (see Fig. 1b). This technological solution is selected for several purposes: first, the very small amount of synthesized crystals (a maximum quantity of $1 \mathrm{mg}$ could be obtained in a hypothetic integral filling of the microchannel) is not suitable for ex situ analyses such as ${ }^{13} \mathrm{C}$ solid state NMR in natural abundance. As a consequence, it is of paramount importance to characterize the crystals directly inside the microchannel. Secondly, the reversible sealing assembly is not limited to standard materials (PDMS, flat glass or silicon) to close the microchannel on the bottom part. Microscope glass slides have been selected for X-ray diffraction (XRD) analysis and for observations using field emission scanning electron microscopy (SEM) as well as for Raman scattering experiments. Although
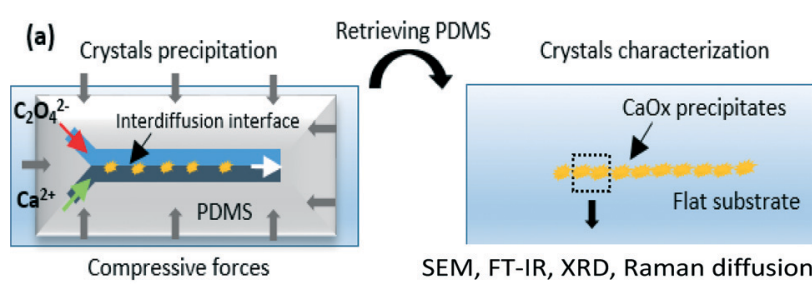

Compressive forces

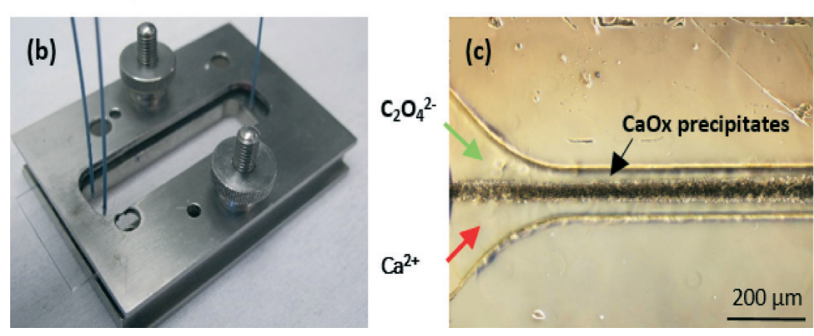

Fig. 1 a) Principle of the dismountable two-phase laminar flow microfluidic device used for the synthesis and characterization of precipitated $\mathrm{CaOx}$ crystals. b) Image of the microfluidic device assembled within the compressive metallic holder. c) Optical micrograph showing the "conic shape" precipitation of $\mathrm{CaOx}$ crystals at the dynamic interface in the zone of molecular interdiffusion. coupling these techniques to microfluidic systems have been described separately using different geometries and materials due to the requirements of each technique, ${ }^{12}$ herein the reversibility of our microfluidic system allows us to undergo different characterizations on the same channel as well as the same sample without any technical limitations, thus avoiding the problems of reproducibility from one channel to another.

To initiate the precipitation of the CaOx crystals, the two inlets of the microchannel were filled with a calcium chloride aqueous solution at $12.0 \mathrm{mmol} \mathrm{\textrm {L } ^ { - 1 }}$ and a sodium oxalate aqueous solution at $0.40 \mathrm{mmol} \mathrm{L}^{-1}$, respectively. These concentrations correspond to hypercalciuria and moderate hyperoxaluria, which are frequent settings in kidney stone formers and relate statistically to the presence of both COM and COD phases in patient urine and stones. ${ }^{3 c}$ Two syringe pumps (Harvard apparatus, Pico 11 plus) were used to inject both precursors with flow rates set to $1 \mu \mathrm{min}^{-1}$. After 2 hours at room temperature $\left(22^{\circ} \mathrm{C}\right)$, a dark conic shaped film appeared at the dynamic interface in the zone of molecular interdiffusion. Precipitation progressively increased toward the outlet (see Fig. 1c). At the end of the experiment, both parts of the microchannel are retrieved from the metallic holder. The glass slide support (with the CaOx crystals) is rinsed thoroughly with deionized water and ethanol. Finally, the slides were stored at $4{ }^{\circ} \mathrm{C}$ to limit the transformation of the unstable COD phase into the stable COM phase.

SEM observations performed on the channel walls such as in Fig. 2 show different crystal shapes and sizes (ranging from 1 to $20 \mu \mathrm{m}$ ), depending on the lateral position (perpendicular to the flow) in the precipitation cone. Trying to rationalize qualitatively these observations, the concentration of each precursor and the resulting SS (see ESI $\uparrow$ for the definition and Fig. S1-S3) gradients established due to diffusion under laminar flow in the microchannel were calculated using COMSOL multiphysics. Nucleation and growth of $\mathrm{CaOx}$ involve homogeneous and heterogeneous phenomena and have been largely studied in different media including

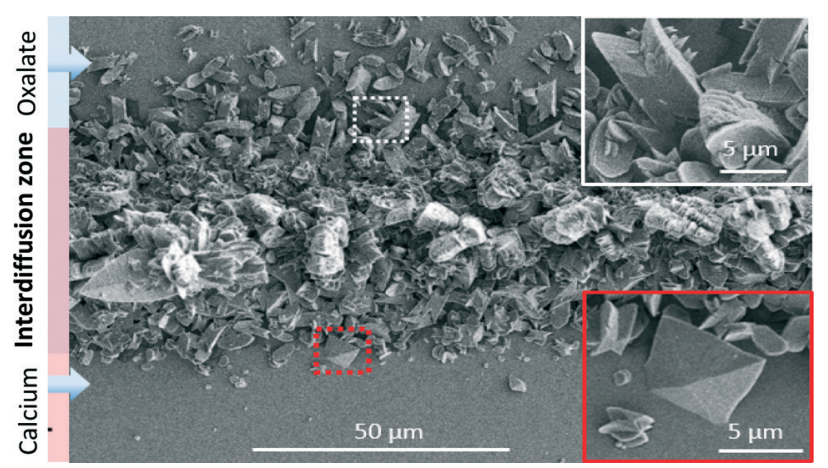

Fig. 2 SEM image of precipitated crystals obtained in the microfluidic device from oxalate and calcium solutions at the dynamic interface. The white inset shows a zoom of the upper part of the precipitate containing twinned crystals. The red inset shows a zoom of the lower part of the precipitate containing mixed twinned and tapered bipyramid crystals. 

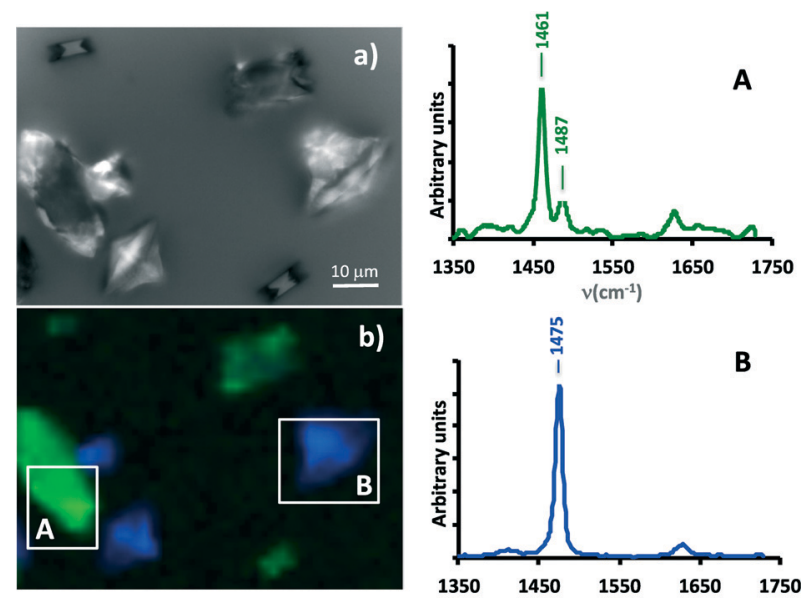

Fig. 3 a) Optical image of a portion of the microchannel, and b) corresponding Raman mapping for two spectral regions, 1452-1468 $\mathrm{cm}^{-1}$ in green and $1470-1480 \mathrm{~cm}^{-1}$ in blue. The Raman spectra A and B correspond to the A and B zones indicated in b).

artificial and natural urine. ${ }^{13}$ It is well accepted that the driving force of CaOx nucleation is the SS. When SS $\gg 1$ and in the zone of unstable SS, CaOx nucleates and grows. ${ }^{13 b}$ As can be seen in Fig. 2 the biggest crystals of CaOx are located at the centre of the precipitate, i.e. in the middle of the channel where the SS is at a maximum (ESI, † Fig. S3). From the center to each edge, a gradual decrease of crystal sizes is observed which is correlated as well to a progressive decrease of oxalate (or calcium) concentrations and consequently of SS. Outside the precipitation cone, no nucleation is observed because the supersaturation regime was not reached. In the upper region of the image (rich in oxalate), a majority of twinned crystals representative for the COM phase were observed (see white inset in Fig. 2) ${ }^{14}$ In terms of physiology, this condition corresponds to a low calciuria with a moderate hyperoxaluria inducing more frequently the predominant occurrence of the COM phase in patient stones. ${ }^{3 c}$ In the lower region of the image bipyramids (representative for the COD phase), were additionally observed. ${ }^{15}$ At that location, the oxalate concentration is lower than the calcium concentration, which physiologically corresponds to hypercalciuria with a low oxaluria inducing indeed the presence of the COD phase in patient urine stones more frequently. ${ }^{3 c}$

XRD diffraction experiments were performed on the precipitate obtained on a microscope glass slide, which is not the best suited substrate due to high scattering: only the COM phase was clearly identified as presented in ESI $\dagger$ (Fig. S4). ATR FT-IR spectra of the precipitated crystals were also recorded in the upper and lower regions of the microchannel (see Fig. S5 in ESI + ): the resulting spectra confirm the presence of the COM phase in the upper region, but fail to clearly identify the COD phase. Indeed, the identification of COM and COD in a mixture has been reported to be difficult to establish by FT-IR, especially when one compound (COM) is in excess. ${ }^{16}$ Furthermore, the low signal to noise in- herent with the ATR-FT-IR imaging system that was used didn't help for this purpose.

We thus decided to rely on Raman scattering experiments to distinguish COM and COD phases, which is rather straightforward with this spectroscopy since COM is characterized by two Raman bands at 1463 and $1490 \mathrm{~cm}^{-1}$, while COD presents only one Raman band in this frequency range, at $1472 \mathrm{~cm}^{-1} \cdot{ }^{17}$ A portion of the microchannel where the two crystal morphologies found by SEM could be seen (Fig. 3a) was imaged thanks to a Raman microscope (Fig. 3b). The two types of crystals, twinned crystals (A zone) and bipyramids (B zone) indeed correspond to two different Raman spectral responses that are in total agreement with COM and COD phases, respectively. Raman mapping is indeed a powerful technique to distinguish between the two CaOx phases present in the microchannel and definitely strengthen the interpretation proposed from the SEM experiments.

In this work, the effect of mixing by interdiffusion on calcium oxalate crystalline phases from calcium and oxalate precursors at miscible liquid interfaces and under physiological conditions (concentrations and flow rates) was studied using a microfluidic channel. Such a channel had similar dimensions as the collection duct in human kidneys and acted as a biomimetic model in order to understand the formation of pathological microcalcifications. Moreover due to the versatility of the microsystem, structural characterizations on the CaOx crystals in the microchannel could be performed using SEM, ATR FT-IR, Raman diffusion and XRD techniques. In agreement with observations related to urinary lithiasis in patients, the variation in oxalate and calcium concentrations along the lateral section of the microchannel oriented the preferential formation of COM or COD phases. These preliminary results highlighted the potential of microfluidic devices to obtain crystalline phases relevant to human pathological calcifications. Moreover, our microfluidic approach opens up new opportunities to study the mechanisms leading to the first steps of kidney stone formation in collecting ducts. In the near future, the influence of various physico-chemical parameters such as the role of biological fluids (urea, ionic salts...) and growth inhibitors (citrate, catechine...) will be investigated quantitatively.

\section{Acknowledgements}

This work was supported by French state funds managed by the ANR within the Investissements d'Avenir programme (ANR-11-IDEX-0004-02), and more specifically within the framework of the Cluster of Excellence MATISSE led by Sorbonne Universités. The authors acknowledge LabEx MICHEM (Sorbonne Universités) for the use of nano/microRaman facilities.

\section{References}

1 V. Romero, H. Akpinar and D. G. Assimos, Rev. Urol., 2010, 12, e86-e96. 
2 E. M. Worcester and F. L. Coe, N. Engl. J. Med., 2010, 363, 954-963.

3 (a) F. Grases, A. Costa-Bauza, I. Gomila, M. Ramis, A. GarciaRaja and R. M. Prieto, Urol. Res., 2012, 40, 41-46; (b) D. Milošević, D. Batinić, P. Konjevoda, N. Blau, N. Štambuk, L. Nižić, K. Vrljičak and D. Batinić, J. Chem. Inf. Model., 2003, 43, 1844-1847; (c) M. Daudon, P. Jungers and O. Traxer, Lithiase urinaire, Medecine Sciences Publications, Paris, 2012.

4 (a) J. P. Kavanagh, Urol. Res., 2006, 34, 139-145; (b) B. Bohner, G. Schuszter, O. Berkesi, D. Horvath and A. Toth, Chem. Commun., 2014, 50, 4289-4291.

5 J. F. Bertram, R. N. Douglas-Denton, B. Diouf, M. D. Hughson and W. E. Hoy, Pediatr. Nephrol., 2011, 26, 1529-1533.

6 P. D. Vize, A. S. Woolf and J. B. L. Bard, The kidney: from normal development to congenital abnormalities, Elsevier Science/Academic Press, Amsterdam, 2003.

7 P. Tabeling, Introduction to Microfluidics, Oxford University Press, Reprint edition, 2010.

8 (a) P. J. A. Kenis, R. F. Ismagilov and G. M. Whitesides, Science, 1999, 285, 83-85; (b) A. Yashina, F. Meldrum and A. deMello, Biomicrofluidics, 2012, 6.

9 S. N. Bhatia and D. E. Ingber, Nat. Biotechnol., 2014, 32, 760-772.
10 Z. Wei, P. K. Amponsah, M. Al-Shatti, Z. Nie and B. C. Bandyopadhyay, Lab Chip, 2012, 12, 4037-4040.

11 A. Konda, J. M. Taylor, M. A. Stoller and S. A. Morin, Lab Chip, 2015, 15, 2009-2017.

12 (a) M. V. Barich and A. T. Krummel, Anal. Chem., 2013, 85, 10000-10003; (b) R. Barrett, M. Faucon, J. Lopez, G. Cristobal, F. Destremaut, A. Dodge, P. Guillot, P. Laval, C. Masselon and J. B. Salmon, Lab Chip, 2006, 6, 494-499; (c) A. F. Chrimes, K. Khoshmanesh, P. R. Stoddart, A. Mitchell and K. Kalantar-zadeh, Chem. Soc. Rev., 2013, 42, 5880-5906; (d) B. Lehmkuhl, S. D. Noblitt, A. T. Krummel and C. S. Henry, Lab Chip, 2015, 15, 4364-4368.

13 (a) F. J. Opalko, J. H. Adair and S. R. Khan, J. Cryst. Growth, 1997, 181, 410-417; (b) B. Finlayson, Kidney Int., 1978, 13, 344-360; (c) J. M. Baumann and B. Affolter, World J. Nephrol. Urol., 2014, 3, 256-267.

14 A. Millan, Cryst. Growth Des., 2001, 1, 245-254.

15 R. C. Walton, J. P. Kavanagh, B. R. Heywood and P. N. Rao, J. Cryst. Growth, 2005, 284, 517-529.

16 P. Carmona, J. Bellanato and E. Escolar, Biospectroscopy, 1997, 3, 331-346.

17 C. Conti, M. Casati, C. Colombo, E. Possenti, M. Realini, G. D. Gatta, M. Merlini, L. Brambilla and G. Zerbi, Spectrochim. Acta, Part A, 2015, 150, 721-730. 